\title{
IKERLANAK
}

\section{MINORITY LANGUAGE AND THE STABILITY OF BILINGUAL EQUILIBRIA}

by

Nagore Iriberri and José Ramón Uriarte

2011

Working Paper Series: IL. 45/11

Departamento de Fundamentos del Análisis Económico I

Ekonomi Analisiaren Oinarriak I Saila



University of the Basque Country 


\section{Minority Language and the Stability of Bilingual Equilibria Nagore Iriberri** \\ Universitat Pompeu Fabra \\ Departament d'Economia i Empresa \\ 08005 Barcelona \\ José Ramón Uriarte** \\ Universidad del Pais Vasco-Euskal Herriko Unibertsitatea \\ Departamento de Fundamentos del Análisis Económico I \\ Ekonomi Analisiaren Oinarriak I Saila \\ 48015 Bilbao}

December 2010

* We are grateful to Ehud Kalai, Ignacio Palacios-Huerta, Karl Schlag, Larry Samuelson, Reinhard Selten, Joel Sobel and Bengt-Arne Wickström for their comments and criticisms to different versions of this work.

** Nagore Iriberri acknowledges financial support from Ministerio de Educación y Ciencia (SEJ2006-05455 and SEJ2007-64340) and the support of the Barcelona GSE Research Network and of the Government of Catalonia. José Ramón Uriarte gratefully acknowledges the financial support from the Spanish Ministry of Science and Technology and FEDER, grant number SEJ 2006-05455, and the University of the Basque Country, UPV 00043.321-15836/2004. The usual disclaimer applies.

JEL Classification Numbers: C72, D81. Keywords: Imperfect Information, Majority/Minority Language, Language Competition.

Corresponding author: J.R. Uriarte, University of the Basque Country, Facultad de Ciencias Económicas y EE. Departamento FAE I-EAO 1S, Avenida del Lehendakari Agirre, 83, 48015 Bilbao, Basque Country-Spain. E-mail: jr.uriarte@ehu.es. T:0034 946013778. 


\begin{abstract}
We investigate a society with two official languages: $A$, shared by all individuals and $B$, spoken by a bilingual minority. Thus, it is only $B$ that needs to increase its population share, and therefore, only the language dynamics that derive from the interactions that occur inside the bilingual population are both empirically and theoretically relevant. To this end, a model is developed in which the bilingual agents must make strategic decisions about the language to be used in a conversation. Decisions are taken under imperfect information about the linguistic type of the participants in the interaction. We first study all the possible equilibria the model might produce and the language used in each of them. Then, in a dynamic setting, we study the building of a language convention by the bilingual speakers.

The main result is that there is a mixed strategy Nash equilibrium in which bilingual agents use both the $A$ and $B$ languages. This equilibrium is evolutionary stable, and dynamically, it is asymptotically stable for the one-population replicator dynamics. In this equilibrium, the use of $B$ between bilingual individuals could be very low.
\end{abstract}




\section{Introduction}

Abrams and Strogatz (2003) proposed a model for the dynamics of language death which has triggered a burst of research on language competition and diversity (e.g. Patriarca and Leppänen, 2004; Pinasco and Romanelli, 2006; Mira and Paredes, 2005; Stauffer and Schulze, 2005; Wang and Minnet, 2005, 2008; Castelló et al. 2006; Stauffer et al. 2007). The model deals with two languages that compete with each other for speakers and predicts that there is no stable coexistence of the two languages; one will eventually drive the other to extintion. As noted by Wang and Minnet (2005), a significant weakness of this model is that only monolingual speakers of $A$ and $B$ are taken into account.

Of course, bilingual societies do exist and, in most of them, what we find is an asymmetric language competition. Typically, what we would have is a society in which there are two languages, one, denoted $A$, that has (historically) achieved that its speech community be the whole society, and the other, denoted $B$, that is spoken by just a bilingual minority (the $A$ and $B$ speakers). Often, $B$ is an endangered language which, to avoid extintion, must increase its speaker population both by converting some of the $A$ monolingual speakers into bilingual speakers and by ensuring the intergenerational transmission through a bilingual educational system.

This is the case of Welsh and Scottish Gaelic competing with English; the Basque language competing with French and Spanish; Breton, Catalan and the Occitan languages (Gascon, Provençal, Aranés ) competing with French; Sami competing with Swedish, Norwegian and Russian; Frisian, spoken in the province of Friesland in The Netherlands, competing with Dutch; the Aboriginal languages of New Zealand and Australia competing with English; Native American languages (Quechua, Aimara, Guarani, among others) competing with English, Spanish, French, Portuguese and Dutch; languages from the republics of Russia competing with Russian. See Fishman (2001) for more examples.

Essentially, in all those cases, it is only $B$ that needs to increase its population share and expand its use to every social domain. Therefore, only the language dynamics that derive from the interactions that occur inside the bilingual population are both empirically and theoretically relevant.

In the present paper, we shall assume a society whose constitution states that $A$ and $B$ are the official languages of the community, and that they should have equal rights, be equally used and promoted. In this society every individual speaks $A$ and a minority speaks both $A$ and $B$. Thus, we 
have just two (linguistic) groups: the $A$ monoglots and the bilingual minority.

Our view is that the asymmetric competition between $A$ and $B$ cannot be properly described by means of models based on the assumption that language attractiveness increases monotonically with the proportion of its speakers and its status ( Abrams and Strogatz, 2003, Minnet and Wang, 2008): the attractiveness of $A$ would always dominate that of $B$, and, the status (that is, the expected economic and social opportunities to the speakers) of $A$ in the community, would also be much higher than that of $B$ (for asymmetric attractiveness, see Pinasco and Romanelli, 2006). The study of this competitive situation would require of models describing the language decisions taken by the bilingual individuals when they interact. Typically, decisions concerning the use (and the learning) of $B$ are guided by a mixture of loyalty to the language and its related culture, a sense of belonging to the community that supports that language, socially induced cultural habits, and also a consideration of the practical advantages one would get (Bengt-Arne, 2005).

The consensus among language planners and sociolinguists is that a minimal condition for the survival of a minority language is that it be spoken by its speech community (see, e.g., Crystal, 2001, Fishman, 2001, Krauss, 1992 and Wurm, 2001). Hence, the model that we propose looks into the language-usage that bilingual speakers make of $B$.

We view the bilingual population not uniformly distributed, but as an influential population in some communities within the large network of the society under study; for instance, in certain geographical areas of the country (Patriarca and Leppänen, 2004). Inside one of those communities there will exist dense internal links, and, frequently, $B$ will be the language used as a means of communication. The internal interactions, repeated through time, will generate almost a perfect information about the language knowledge of the members of the community. But between the communities (of any nature, not just those where the bilingual population is proportionally important) links are less dense and, jointly with the fact that the perfect knowledge about the bilingual or monolingual nature of who you are interacting with is lost, the use of B would be drastically reduced. This is one of the biggest issues that endangered languages encounter; that is, the difficulty to increase the density and variety of the language-use beyond the traditional domains (linked to old social organization and economic activities) developed inside their historical strongholds. (See, e.g. Crystal, 2001).

We study precisely the kind of interactions that occur outside the tra- 
ditional communities That is, interactions that might happen in "modern" domains, -that is, urban activities, say, justice administration, media, modern technologies, high education, research and development, and so on-, where, competition with (an adapted to every use; i.e. a normalized) $A$ is harder and where, frequently, individuals are led to meet and interact with people whose bilingual or monolingual nature is unknown (ex-ante). This, we think, is a more plausible situation. We should not forget that there is no language contact without social conflict (e.g. Nelde, 1997 and Grin, 2003). Thus, in many real situations marking or labeling people to reveal their monolingual or bilingual type is (or may be) seen as a potential source of further political conflict. Therefore, in most one-time interaction with public and private administration, agents are not able to recognize others' language knowledge. Further, analytically imperfect information is more relevant than perfect information because, under the assumptions of our model, bilingual individuals with perfect information will coordinate in $B$ (as it happens frequently in real situations). In this setting, we assume that none of the two interacting individuals has or sends any signal that might inform about his/her bilingual or monolingual nature.

We model (the initial steps of) the conversation that takes place in those interactions by means of a non-cooperative game of imperfect information played by two individuals, -called the Language Game. We seek first to know under which strategic conditions language $B$ would be used in a conversations held in those kind of interactions. The paper addresses too the role of the strategic behaviour of bilingual agents in stabilizing Nash equilibria where both A and B are used; that is, we want to know the speech conventions built by the bilingual population. The paper seeks to give an answer to the question posed by Wickstrom (2005), which in the context of our model would be, is there a Nash bilingual equilibrium dynamically stable? Similarly, Castelló et al. (2006), using different tools, deal as well with the issues of the stabilizing role of the bilingual agents and that of the emerging of (linguistic) norms or conventions.

Of course, imperfect information reduces considerably the use of the minority language, aggravating its minority status. It can be seen that this is independent of the length of the introduction to the conversation modelled by the language game. Nevertheless, we show that there is a bilingual equilibrium, - a bayesian-perfect mixed strategy equilibrium in which both $A$ and $B$ are used by the bilingual population-, that is evolutionary stable. This means that any small group of bilinguals ("mutants") who experiment 
with an alternative strategy, -including $B$-monolingual strategies - will do less well than those bilingual agents attached to the incumbent mixed strategy. Therefore this mixed equilibrium would be the convention used by the bilingual agents in interactions that occurred under imperfect information. We also show that this equilibrium is dynamically stable for the one population replicator dynamics. On the negative side, it should be stressed that this robust equilibrium is compatible with bilingual agents playing language strategies where the use of $B$ is with a probability close to zero. We conjecture that that situation might actually happen when bilingual strategies are chosen under the influence of the principles of politeness (Lakoff, 1973 and Brown and Levinson, 1987). Therefore, we cannot conclude that the survival of $B$ outside the traditional activities, - that is, in those where $A$ or stronger languages are dominant and interactions are frequently between anonymous agents -, is guaranteed.

Mira and Paredes (2005), show that the key factor for the stability of bilingualism is the interlinguistic similarity of $A$ and $B$; and Patriarca and Leppänen (2004) show that $A$ and $B$ may coexist if they are concentrated in disjoint geographical areas. We instead prove the stability of a bilingual equilibrium assuming that $A$ and $B$ are linguistically distant, so that a conversation is only possible in one language; and, as mentioned above, we avoid one-shot interactions taking place in the stronghold areas of $B$.

The paper is organized as follows. In section 2 we present a detailed description of the Language Game in its extensive form and carry out the equilibrium analysis. Section 3 deals with the building of a speech convention, and presents the main result.

\section{The Language Game}

The Language Game captures a simple social interaction between two agents in which a, so-called, shopkeeper starts a conversation with a, socalled, potential buyer. This interaction takes place in a society with two official languages (so that the language choice is not trivial): language $A$, called majority language, spoken by every individual in the society, and language $B$, called minority language, spoken by a relatively small proportion of individuals and having a small range of social usage. Thus, even though by law both languages have the same legal status, the weaker $B$ must compete for speakers and for the same social functions as $A$. There are two types of individuals in the society: the bilingual type, who speaks both $A$ and $B$ and therefore can choose over the two possible actions or languages 
$\left(a_{b i}=\{A, B\}\right)$; and the monolingual type, who only speaks the majority language $A$ and therefore has no language choice $\left(a_{m o}=\{A\}\right)$. Let $\alpha$ and $(1-\alpha)$ denote the proportion of bilingual and monolingual individuals, respectively. We assume that $\alpha<(1-\alpha)$; it is also assumed that $\alpha$ is much smaller than $(1-\alpha)$.

Types are privately known, but the distribution of types in the society is publicly known. In other words, each individual knows her own type, whether she is bilingual or monolingual, and also that the proportion of bilingual and monolingual individuals in the society is $\alpha$ and $(1-\alpha)$, respectively.

Assumption 1: The linguistic distance (see Crystal (1987)) between $A$ and $B$ is sufficiently high so that successful communication is only possible when the interaction takes place in one language.

This assumption is important to understand that the language choice is not a trivial one. In other words, it is not possible to have a conversation where one individual speaks $A$ and the other one $B$ because a monolingual agent would not be able to understand what is being said when someone uses language $B$. This also implies that when a monolingual interacts with a bilingual the interaction will necessarily take place in the majority language $A$. Notice that this assumption might not be realistic in, say, some european regions, such as Galicia (northwest Spain) where it is possible to have a conversation in which one agent speaks $A$ (Spanish) and the other replies using $B$ (Galician), because the linguistic distance between $A$ and $B$ is not too big (i.e. both are romanic languages). However, in other regions in which two official languages are in contact, such as in the Basque Country, mixed language conversations are not common because the linguistic distance between $A$ (Spanish or French) and $B$ (Basque) is big enough (Basque is a preindoeuropean language).

The following assumptions think of bilingual individuals as language $B$ loyal agents.

Assumption 2: Bilingual players prefer to speak $B$ rather than $A$. Formally, let $\succ_{b i}$ denote the preference relation of a bilingual agent, such that $B \succ_{b i} A$.

Two reasons could justify that preference relation. Pool (1986) intro- 
duced a game with perfect information and only bilingual players, where each player's native language is the other's second language, and assumed that both players prefer to speak their own native language. Along the same line, we could interpret this assumption as considering that all bilingual players' native language is $B .{ }^{1}$ Also, the culturally specific language of any society "is more than just a tool of communication for its culture. (...). Such a language is often viewed as a very specific gift, a marker of identity and a specific responsibility vis-à-vis future generations" (Fishman (1991)). This is a statement valid for any language, but the minority condition of language $B$ strengthens this conception. Bilingual players are aware that $B$ is a minority language and therefore an endangered language. They consider that the only way to avoid its disappearance is by using it, such that, whenever possible, they have a preference for using $B$ over $A$. Monolingual individuals have no choice and so no preference for a language over the other.

Before we present the third assumption, which is about the payoff ordering, notice that there are three payoff relevant situations in the language game. First, bilingual players might coordinate on their preferred language $B$. In that case, we will assume both players get payoffs equal to $m$. Second, either bilingual or monolingual players might coordinate on the majority language $A$. In that case, we will assume both players get payoffs equal to $n$, because this was a voluntary coordination or choice. Finally, a bilingual player might try to coordinate on her preferred language $B$ but fail to do so ending using the majority language $A$. In this latter case, the interaction will take place in the majority language $A$, and we will assume that the bilingual or monolingual player who chose $A$ will get a payoff of $n$, while the bilingual player who tried to use $B$ will get a payoff of $n-c$, (where $c$ represents the frustration or disenfranchisement cost) due to the forced change in the use of language, from $B$ to $A$ ). Notice that, since $n$ is the payoff that a bilingual player can obtain for sure had he chosen to speak $A$, the frustration cost $c$ should be subtracted from $n$. Thus, $(n-c)$ is the payoff to a bilingual player who, having chosen $B$, is matched to someone, monolingual (or bilingual), who uses language $A$ and, therefore, must end up speaking $A$. Given the three payoff relevant situations, we will now order the three payoffs in the following assumption.

\footnotetext{
${ }^{1}$ This assumption can be relaxed, where the bilingual types would be further divided into two different types, some might have the ordering assumed as in Assumption 2 and some other might have the opposite ordering. This is a possible extension but does not affect the main result we show in this paper.
} 
Assumption 3: For a given $\alpha$ such that $\alpha<(1-\alpha)$, the payoff ordering is given by $m>n>c>0$.

The first inequality ${ }^{2}, m>n$, is given by Assumption 2, which must be interpreted as bilingual players preferring $B$ to $A$, such that they will get a higher von Neumann and Morgenstern utility when they interact in their preferred language $B$ than in the case when they choose to use $A$. "Switching to a minority language is very common as a mean of expressing solidarity with a social group. The change signals to the listener that the speaker is from a certain background; and if the listener responds with a similar switch, a degree of rapport is established" (Crystal (1987), Chapter 60). The cost, $c$, which we assume to be smaller than $n$, is intended to capture the dissatisfaction, mentioned by Fishman (1991), felt by the bilingual player who must face the fact that, in many interactions, he is forced to use the majority language $A$. Following Fishman (1991), the efforts made by the bilingual population to reverse language shift are an indication of dissatisfaction with the cultural life which is dominated by the majority language.

\subsection{The Language Game with Imperfect Information}

The players know their own type, whether they are bilingual or monolingual, but do not observe other players' type. What they do know is that both speak the majority language $A$ and that only a minority speaks $B$, as well as the proportion of bilingual $(\alpha)$ and monolingual $(1-\alpha)$ individuals in the society. This setting represents the interaction among individuals who do not know each other and therefore each others' bilingual or monolingual nature, such as it occurs in many real life situations. As mentioned in the introduction, to our knowledge, the existence of imperfect information and its consequences with regard to the use of the minority language has never been stressed in the literature dealing with minority languages. The case of perfect information is trivial: two bilingual players would meet with probability $\alpha^{2}$, and would coordinate in their preferred language $B$, hence, maximizing their payoffs .

We will proceed in three steps. First, we will describe the extensive form game under imperfect information, then we will carry the equilibrium

\footnotetext{
${ }^{2}$ In section 3 the payoffs are thought to be sensitive to $\alpha$.
} 
analysis. Finally, we present the evolutionary dynamics setting that will select among the multiple Bayesian Perfect Nash equilibria.

\subsubsection{The Description of the Game}

The social interaction where a shopkeeper (player I) and a potential buyer (player II) choose a language for having an effective communication is shown in Figure 1. It is an extensive form game where actions are taken sequentially, as in an ordinary conversation, so that one player starts the conversation and the other player replies (an equivalent representation of the game is given in Figure 3, in the Appendix). A bilingual shopkeeper starts the interaction using one of the two languages. On the one hand, if the shopkeeper chooses $B$ the potential buyer can recognize the shopkeeper is bilingual and can reply using $A$ or $B$ if bilingual or using $A$ if monolingual. On the other hand, if the shopkeeper chooses $A$ the potential buyer cannot distinguish whether the shopkeeper is bilingual or monolingual and she can reply using $A$ or $B$ if bilingual and using $A$ if monolingual. If the potential buyer replies with $B$ after hearing $A$, the shopkeeper has a second chance to change or not her initial choice of language.

The key aspect of this game is that the knowledge of each player about the type of the other player is imperfect. The presence of Nature represents the unawareness of each player about the other player's type. Nature chooses bilingual players with probability $\alpha$ and monolingual players with $(1-\alpha)$. As it is described in the tree, the second player can observe (hear) the language choice but not the type. As a consequence, $B$ will be used if both players happen to be bilingual and happen to coordinate in $B$. In any other case, the majority language will be used. For example, when both players are bilingual but coordinate in $A$ or when a bilingual player is matched to a monolingual player, independently of the choice of language made by the bilingual player, the interaction will necessarily take place in language $A$. Given the monolingual type has no proper choice to make, we will only look at bilingual players' information sets and strategies.

In Figure 1, we can see a bilingual player I has two information sets called Bilingual Ia and Bilingual Ib. Bilingual Ia: player I knows she is bilingual, and thus at this information set she must choose among the languages $A$ or $B$ in order to start a conversation with player II, whose type she cannot observe. Hence, player I is uninformed at this information set. Bilingual $I b$ : this information set starts a proper subgame and player I chooses after player II has revealed her bilingual nature. Thus, player I is informed in this 


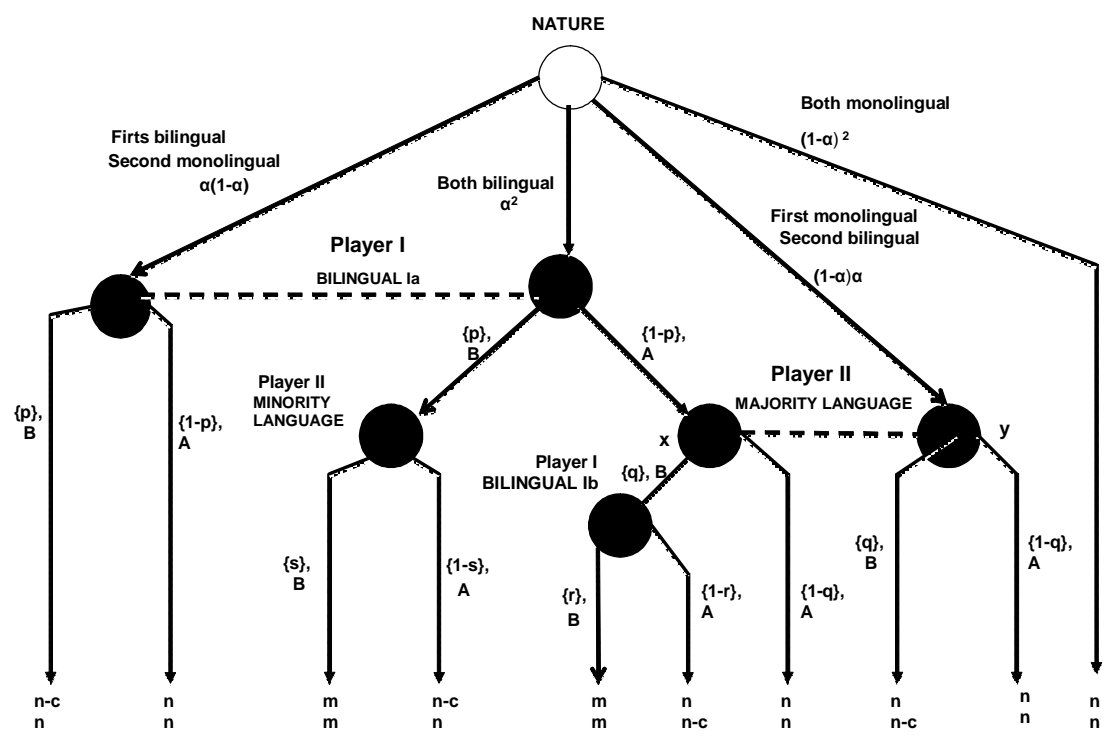

Figure 1: The Language Game, where A denotes the majority language, B the minority language, c the "frustration" cost, and $\alpha$ and 1- $\alpha$ the proportion of bilingual and monolingual individuals, respectively in the society. The probability with which an action is chosen is given by $\{$.$\} .$

information set. The bilingual player II has also two information sets, which we will refer to as Minority Language and Majority Language information sets. Minority Language (MiL): this information set starts a proper subgame. Here, player II may choose after player I has revealed her bilingual nature because she decided at Bilingual $I a$ to choose $B$. Hence, player II is informed in this information set. Majority Language (MaL): a bilingual player II makes choices in this set after having heard player I started the conversation using the majority language $A$. In this set, player II cannot observe whether player $\mathrm{I}$ is bilingual or monolingual. Thus, this information set contains two nodes, $x$ and $y$. 
Bilingual player I has four pure strategies, $S_{I}=\{B B, B A, A B, A A\}$. In each strategy, from left to right, the left component is the action taken at the Bilingual $I a$ information set, and the right component is the action taken at her second information set, Bilingual $I b$. Therefore, strategy $B B$ describes a plan where player I always speaks $B$. The second strategy $B A$ represents a plan where player I uses $B$ first and then switches to the language not used by player II. Notice that player I's $B B$ and $B A$ strategies are equivalent because the bilingual player I's information set Bilingual $I b$ is off the path of play and therefore, she will not have the opportunity to reply when player II uses $B$ at Majority Language information set. Third, $A B$ describes the strategy where player I uses $A$ first and then switches to the language spoken by player II. Finally, $A A$ represents a plan where player I always speaks $A$.

Bilingual player II's pure strategy set is $S_{I I}=\{B B, B A, A B, A A\}$. In each strategy, from left to right, the left component is the decision taken at her Minority Language information set and the right component is the action taken at the Majority Language information set after she has heard player I speaking $A$. Strategy $B B$ represents a plan where player II always replies using $B$ regardless of whether she has heard player I using the minority or the majority language. The second strategy $B A$ describes a plan where player II always replies in the language spoken by player I. Third, $A B$ represents a plan where player II replies in the language not used by player I. Finally, $A A$ represents the strategy where player II always replies using $A$.

Finally, it is important to analyze the language in which the social interaction takes place in each possible strategy combination. Each pure strategy profile can be thought of as the initial phase of a conversation between two bilingual players in which the choice of language for the social interaction is determined. As in the case with perfect information, four possible events or combination of player types may occur: with probability $\alpha^{2}$, both players I and II are bilingual, with probability $\alpha(1-\alpha)$ player I is bilingual and player II is monolingual, with probability $(1-\alpha) \alpha$ player I is monolingual and player II is bilingual and, finally, with probability $(1-\alpha)^{2}$ both players are monolingual. The Language Matrix, shown in Figure 2, shows the language associated to each pair of strategies when played by bilingual players, which is the only non-trivial case on which we concentrate. In the rest of the three possible events, since at least one player is monolingual, the spoken language will be $A$. For instance, let us look at that the strategy profile $(A B, A B)$. In this situation, the language that will be used by bilingual players is determined as follows: player I starts choosing $A$ at Bilingual Ia information 
set and player II, after hearing language $A$, replies by choosing $B$ at her Majority Language information set. Then, player I reaches her Bilingual Ib information set and there she switches to $B$. Hence, under this strategy profile, the minority language $B$ will be actually used when the realized event is the matching of two bilingual players.

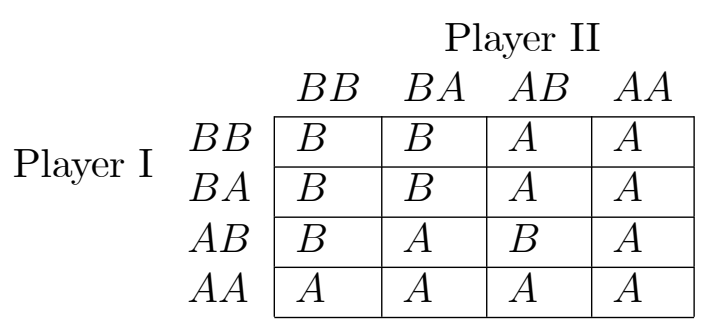

Figure 2. The Language Matrix

The above matrix shows that two bilingual players, which happen to meet with probability $\alpha^{2}$, will be able to coordinate on their preferred language, $B$, only at 6 pure strategy profiles out of 16 possible strategy combinations. In the next section we will analyze the Bayesian Perfect Nash equilibrium profiles and study what imperfect information brings to the use of each of the languages $A$ and $B$.

\subsubsection{When is it Optimal for Bilingual Individuals to Use Lan-} guage $\mathrm{B}$ ?

Recall that the Language Game in Figure 1 has two proper subgames. One subgame starts at player I's Bilingual Ib information set and the other at player II's Minority Language information set. In both subgames, $B$ is the best choice for both players ( i.e. $r=1$ for player I and $s=1$ for player II) because the player who moved previously, by choosing $B$, has revealed that she is bilingual; thus, both players may select the outcome they prefer most. This implies that any equilibria that does not include action $B$ in these two subgames cannot be bayesian-perfect.

A language $B$ loyal agent will take care of not making the mistake of playing the strategies that would clearly block the use of $B$. Thus, we may think that inside the bilingual population there exists a tacit agreement that 
when, in a conversation (under imperfect information), one speaker reveals his bilingual nature by using $B$, the other, -if it happens to be bilingual too-, should answer using language $B$. In the present model, the agreement would imply that player I should choose $B$ at Bilingual Ib information set when this set is reached with a non-zero probability; and, similarly, player II would choose $B$ at his Minority Language set. Hence, a bilingual in the position of player I will avoid to play the strategies $B A$ and $A A$, since they go against the agreement. Similarly, in the position of player II the strategies $A B$ and $A A$ will be avoided. Hence, the pure strategies that a bilingual individual in the position of player I will use are $S_{I}=\{B B, A B\}$ and those for the position of player II are $S_{I I}=\{B B, B A\}$. The combination of these strategies are the candidates to form Bayesian-perfect equilibria. Therefore, the language loyalty behaviour of bilingual individuals coincides with the rational behaviour in the game.

We shall consider three regimes that derive from the assumption that could be made about the possible value of the frustration cost, $c$, felt by bilingual players, vis-a-vis the benefit, $(m-n)$, this type of players might obtain when they are able to use their preferred language. We shall describe first the Bayesian Perfect equilibria in pure strategies ${ }^{3}$. The analysis of mixed strategy equilibria is made in section 3 .

\section{Proposition 1}

Let the proportion of bilingual individuals, $\alpha$, be given, with $\alpha<1-\alpha$.

Regime 1. $\quad c=(m-n) \frac{\alpha}{(1-\alpha)}$; the set of Bayesian Perfect equilibria is: $B P N^{1}=\{(A B, B A),(A B, B B),(B B, B A)\}$

Where $(A B, B A)$ is the only equilibrium with dominant strategies for both players. The language spoken in this equilibrium is $A$.

Regime 2. $c>(m-n) \frac{\alpha}{(1-\alpha)}$; there is only a unique Bayesian-Perfect equilibrium: $B P N^{2}=\{(A B, B A)\}$. The language spoken is $A$.

Regime 3. $c<(m-n) \frac{\alpha}{(1-\alpha)}$; the set of Bayesian-Perfect equilibria is $B P N^{3}=\{(A B, B B),(B B, B A)\}$. The language spoken in both equilibria is $B$.

Proof: see Appendix.

\footnotetext{
${ }^{3}$ In our game Sequential Equilibrium and Perfect Bayesian Equilibrium are equivalent.
} 
Even though bilingual agents are willing to use $B$ and have payoff incentives to do so, Proposition 1 shows that in regimes 1 and 2 they fail to coordinate in the minority language $B$. They use $A$ instead. As one might expect, the proposition shows that the use of minority language $B$ is optimal only in regime 3. But less expected is that the pure equilibria of regime 3 , $(A B, B B),(B B, B A)$, are not good candidates for a language convention for bilingual individuals because they are not evolutionary stable nor dynamically stable. In fact, coordination failures are also present in regime 3 , too. We show this in the next section.

\section{An Evolutionary Stable Bilingual Equilibrium}

Both in regime 1 and regime 2, the model shows that there is a unique bayesian-perfect equilibrium with undominated strategies, $(A B, B A)$ in which $A$ is the spoken language. Thus, the social use of $B$ that would help to guarantee its survival is lowered by the coordination failures that occur due to the choices made under imperfect information, and the risk aversion of the bilingual individuals. The frustration cost $c$ could be said to be a curse for the bilingual agents.

Thus far, we thought of $c$ and $m$ as exogenously given parameters. We may think that steady increases in the proportion of bilingual individuals would increase the use of $B$ and normalize its social use. This process would be, naturally, accompanied by steady reductions of the exceptional costs, $c$, and benefits, $m$, felt by the bilingual people, since $B$ is changing its status of minority language. This would mean that there exists a threshold level $\alpha_{T}$, such that for $\alpha>\alpha_{T}$, both $c$ and $m$ would be decreasing functions of $\alpha$, with $c=c(\alpha)$ approaching 0 and $m=m(\alpha)$ approaching $n$, as $\alpha\left(>\alpha_{T}\right)$ approaches 1 . We shall assume that in that process, $c=c(\alpha)<m=m(\alpha)$ is satisfied.

An example of a minority language community that made the transition from regime 2 to regime 3 , where $c<(m-n) \frac{\alpha}{(1-\alpha)}$, could be the Basque. We should not forget the political dimension of the problem we are dealing with in this paper. During the dictatorial regime that followed the Spanish civil war, the period 1936-1976, the Basque language (in the Basque provinces of Spain) was banned from education and mass media; no public use of the language was allowed. Hence, it could only be spoken in private interactions and its transmission from one generation to the next depended on parental decisions and the influence of the linguistic environment. We may think that 
in that period the discomfort felt by the Basque speakers was high and that the political context made difficult the coordination in $B$ between bilingual individuals unknown to each other. With the restoration of democracy in Spain in 1976, Basque became an official language joint with Spanish. With the aid of a bilingual educational system, the proportion of bilingual (BasqueSpanish) individuals rose from $21.9 \%$ in 1981 to $37.5 \%$ in 2006 (out of a population of around two millions in both years $)^{4}$. The flourishing of TV channels, newspapers and publishing houses increased the output of Basque language related products to satisfy a growing demand. We can safely say that one of the consequences with this new situation is that the frustration cost due to the discomfort felt by being forced to switch from $B$ (Basque) to $A$ (Spanish) was reduced, and that the bilingual community made the transition to regime 3 .

Hence, we can conclude by saying that regime 1, although interesting analytically, is, in fact, unstable.

\subsection{A Bilingual Speech Convention}

In real-life situations, the Language Game described in Figure 1 is not an interaction that occurs once. On the contrary, this game is played many times by many different people and the outcome is not an individual one but a social construct. Therefore, a different analytical approach is needed. This section studies the issue of whether a speech convention based on $B$ could be built. This is equivalent to know if the selected equilibrium from the set of bayesian-perfect Nash equilibria has $B$ as the language used in the interactions between the bilingual agents. To this end, we are led to use an evolutionary approach to complete the equilibrium analysis.

To introduce the evolutionary dynamic setting, let us assume that the bilingual population consists of a large but finite number of individuals who play a certain pure strategy $s_{\mathbf{i}},(i=1,2)$ in a two-player game (see below). The interactions are modelled as pairwise random matching between agents of the bilingual population; that is, no more than two (randomly chosen) individuals interact at a time. The interaction takes place continuously over time. Let $N$ be the total population of bilingual agents in the society and let $x=\frac{\mathbf{N}_{1}}{\mathbf{N}}$ be the proportion of bilingual agents playing pure strategy $s_{\mathbf{1}}$ at

\footnotetext{
${ }^{4}$ The data refer to only the population of the provinces of Biscay, Gipuzkoa and Araba. No data from the provinces of the french side of the Basque Country-Pays Basque nor those from Navarra are included. See, E.J.-G.V. (2009).
} 
any point $t$ in time (time dependence is suppressed in the notation). In this evolutionary setting, a mixed strategy is interpreted as a population state that indicates the population share of agents playing each pure strategy.

\section{Proposition 2}

There exists a symmetric mixed strategy Nash equilibrium $(x, x)=(1-$ $\left.\frac{c(1-\alpha)}{\alpha m}, 1-\frac{c(1-\alpha)}{\alpha m}\right)$ that is an evolutionary stable strategy (i.e., a speech convention) and asymptotically stable in the associated one-population replicator dynamics.

Proof: As we said above, bilingual individuals play with non-zero probability only the pure strategies in the set $\{B B, A B\}$ for player I and those in $\{B B, B A\}$ for player II. If we rephrase these pure strategies, they are, irrespective of the player position a bilingual agent is playing, just the following two types of instructions:

$\mathbf{s}_{\mathbf{1}}$ : Use always $B$, whether you know for certain you are speaking to a bilingual individual or not. That is, play always strategy $B B$.

$\mathbf{s}_{\mathbf{2}}$ : Use $B$ only when you know for certain that you are speaking to a bilingual individual; use $A$, otherwise. This is strategy $A B$ if you start the conversation or strategy $B A$ if you follow the conversation.

Hence, we can say that the agents of the bilingual population play the language game in regime 3 having $S=\left\{\mathbf{s}_{\mathbf{1}}, \mathbf{s}_{\mathbf{2}}\right\}$ as their common strategy set. No information would be lost if we study the bayesian-perfect equilibria of the language game restricted to these two strategies. Then the expected payoff matrix of the language game played by the bilingual agents is the following (we assume that $n=0$ ):

\begin{tabular}{|c|c|c|}
\hline & $\mathbf{S}_{1}$ & $\mathbf{S}_{2}$ \\
\hline & $\alpha m-c(1-\alpha), \alpha m-c(1-\alpha)$ & $\alpha m-c(1-\alpha), \alpha m$ \\
\hline & $\alpha m, \alpha m-c(1-\alpha)$ & 0,0 \\
\hline
\end{tabular}

It can be seen that this is a symmetric (two-player) game. Let us normalize payoffs as follows (see Weibull, 1995, p29):

$$
\begin{aligned}
& a_{1}=a_{11}-a_{21}=\alpha m-c(1-\alpha)-\alpha m=-c(1-\alpha)<0 \\
& a_{2}=a_{22}-a_{12}=0-[\alpha m-c(1-\alpha)]=-\alpha m+c(1-\alpha)<0 \\
& b_{1}=b_{11}-b_{12}=\alpha m-c(1-\alpha)-\alpha m=-c(1-\alpha)<0 \\
& b_{2}=b_{22}-b_{21}=0-[\alpha m-c(1-\alpha)]=-\alpha m-c(1-\alpha)<0
\end{aligned}
$$




\begin{tabular}{|c|c|c|}
\hline & $\mathbf{S}_{1}$ & $\mathbf{S}_{2}$ \\
\hline 1 & $-c(1-\alpha),-c(1-\alpha)$ & 0,0 \\
\hline 2 & 0,0 & $-\alpha m+c(1-\alpha),-\alpha m+c(1-\alpha)$ \\
\hline
\end{tabular}

The game has two asymmetric equilibria, -those corresponding to the perfect equilibria mentioned above, $\left(\mathbf{s}_{\mathbf{2}}, \mathbf{s}_{\mathbf{1}}\right)=(A B, B B)$ and $\left(\mathbf{s}_{\mathbf{1}}, \mathbf{s}_{\mathbf{2}}\right)=$ $(B B, B A)-$, and a symmetric mixed strategy equilibrium:

$$
B P N^{4}=\left\{\left(\mathbf{s}_{\mathbf{2}}, \mathbf{s}_{\mathbf{1}}\right),\left(\mathbf{s}_{\mathbf{1}}, \mathbf{s}_{\mathbf{2}}\right),\left(1-\frac{c(1-\alpha)}{\alpha m}, 1-\frac{c(1-\alpha)}{\alpha m}\right)\right\}
$$

The symmetric equilibrium $\left(1-\frac{c(1-\alpha)}{\alpha m}, 1-\frac{c(1-\alpha)}{\alpha m}\right)$ is an evolutionary stable strategy (that is, a speech convention) and, furthermore, it is an asymptotically stable population state in the single population Replicator Dynamics ( see Proposition 3.10 of Weibull, 1995):

$$
\dot{x}=[\alpha m(1-x)-c(1-\alpha)] x(1-x)
$$

Remark 2: Notice that in regime 3, the language game has the payoff structure of a Hawk-Dove Game (see, e.g., Weibull, 1995). The payoffs of the language game should not be interpreted as biological fitness, but as utility. The replicators are represented here by the pure strategies $\mathbf{s}_{\mathbf{1}}$ and $\mathbf{s}_{\mathbf{2}}$. It is well known that the replicator dynamics could also be derived from behaviours observed in social interactive learning settings; among others, the aspiration-based learning model of Binmore, Gale and Samuelson (1995); or decisions based on imitation and reinforcement of successful behaviour (Schlag, 1998, Weibull, 1995, Cabrales, 2000); or from constant doubts based decision procedures, as in Cabrales and Uriarte (2010). See also Uriarte (2006).

\subsection{Interpretation of the Speech Convention}

(1) We have seen that strategy $\mathbf{s}_{\mathbf{2}}$ is undominated in regime 1 and 2 : $\left(\mathbf{s}_{\mathbf{2}}, \mathbf{s}_{\mathbf{2}}\right)$ is the bayesian-perfect equilibrium in those two regimes. In the evolutionary stable equilibrium of regime 3 there is also a proportion, $\frac{c(1-\alpha)}{\alpha m}$, of bilingual individuals who play strategy $\mathbf{s}_{\mathbf{2}}$. Thus, the model predicts that 
bilingual agents would recurrently use strategy $\mathbf{s}_{\mathbf{2}}$. In real situations one might also expect that this strategy would be widely chosen because, at first sight, it is uncontroversial and satisfies the principles of politeness (of Lakoff, 1973 and Brown and Levinson, 1987). Note that if you start the conversation, $\mathbf{s}_{\mathbf{2}}(=A B)$ advices you to use first the language spoken, not by a minority of the society, but by all the individuals. And if a bilingual receiver answers using $B$, then $\mathbf{s}_{\mathbf{2}}$ advices you to switch to $B$. Therefore, contrary to $\mathbf{s}_{\mathbf{1}}(=B B)$, $\mathbf{s}_{\mathbf{2}}(=A B)$ allows the bilingual listener the option of choosing the language; and a monoglot listener would not feel embarrassed in recognizing her lack of knowledge of $B$, or giving excuses ( such as, she is learning the language and might in the future be fluent in $\mathrm{B}$, but at present is unable). On the other hand, if you were in the position of player II, $\mathbf{s}_{\mathbf{2}}(=B A)$ tells you to use just the language that player I started with.

In other words, $\mathbf{s}_{\mathbf{2}}$ is perceived as non-imposing to monoglots and, by its embodied language switching, allows to choose and use $B$ ( that is, it satisfies the rules of politeness of Lakoff, 1973). Furthermore, it is also perceived as "non face-threatening" (see Goffman, 1967, Brown and Levinson, 1987) to both monolingual and bilingual individuals; it is respectful with the rights and wants of both linguistic groups. Thus, the adoption of strategy $\mathbf{s}_{\mathbf{2}}$ conforms with politeness, which is thought to be basic for social order and cooperation (Brown and Levinson, 1987). Playing $\mathbf{s}_{\mathbf{2}}$, you just avoid a potential conflict.

But in regime 3, the language game has the structure of a Hawk-Dove game. It can be seen in the above payoff matrix, that the conventionally considered unpolite strategy, $\mathbf{s}_{\mathbf{1}}$, is, in fact, a Dove strategy and the conventionally considered polite strategy, $\mathbf{s}_{\mathbf{2}}$, is a Hawk strategy. If both bilingual players behave like doves, they share payoffs and use $B$ in the interaction. If one behaves like a hawk and the other like a dove, the game shows that they will use $B$, but in terms of payoffs, the dove gets smaller expected payoffs because of the risk taken. If both bilingual players behave like hawks, they hurt each other. That is, if they both play strategy $\mathbf{s}_{\mathbf{2}}$, the model shows that the language spoken in the interaction is $A$. Hence, since each thinks the other is monolingual, they do not realize that they are impeding each other their wants of using their preferred language, (see Assumption 2, above) -even though both of them wish to do so- and maximizing their payoffs.

Therefore, the use of the, apparently, polite strategy $\mathbf{s}_{\mathbf{2}}$ is costly for the bilingual population. Thus, in regime 3 , the parameter $c$ could measure the influence of politeness rules on using $\mathbf{s}_{\mathbf{2}}$ by members of the bilingual 
population. Since in regime $3,0<c<m \frac{\alpha}{(1-\alpha)}$; then, for a given $\alpha<1-\alpha$ and $m$, as $c$ approaches $m \frac{\alpha}{(1-\alpha)}$, the proportion $\frac{c(1-\alpha)}{\alpha m}$ of bilingual agents playing $\mathbf{S}_{\mathbf{2}}$ approaches 1 , and therefore, the probability of coordination failure will increase.

Thus, the mixed strategy equilibrium, $\left(1-\frac{c(1-\alpha)}{\alpha m}, 1-\frac{c(1-\alpha)}{\alpha m}\right)$, could be one in which $A$ is dominantly used because the Hawk behaviour is dominant inside the bilingual population. Hence, to conclude, politeness based language conventions could also be an obstruction for the individual and social use of $B$.

(2) This mixed equilibrium could be said to be a bilingual equilibrium because both $\mathbf{s}_{\mathbf{1}}$ and $\mathbf{s}_{\mathbf{2}}$ are played with non-zero probability and hence both $A$ and $B$ will be spoken with non-zero probability.

(3) The mixed strategy Nash equilibrium is robust in the sense that any alternative, pure or mixed, strategy will get smaller payoffs. That is, any small group of bilinguals ("mutants") who experiments with an alternative strategy, -such as a monolingual strategy profile, as in the equilibrium $\left(\mathbf{s}_{\mathbf{2}}, \mathbf{s}_{\mathbf{1}}\right)$ or $\left(\mathbf{s}_{\mathbf{1}}, \mathbf{s}_{\mathbf{2}}\right)$, where only $B$ is used- will do less well than the bilingual agents who stick to the incumbent mixed strategy $\left(1-\frac{c(1-\alpha)}{\alpha m}, 1-\frac{c(1-\alpha)}{\alpha m}\right)$. Therefore, there are no incentives to change the mixed strategy equilibrium, and for that reason, the equilibrium is said to be the speech convention for the bilingual population.

(4). Monolingual equilibria $\left(s_{\mathbf{2}}, s_{\mathbf{1}}\right)$ and $\left(s_{\mathbf{1}}, s_{\mathbf{2}}\right)$ are strong for keeping the language diversity. The non-existence of coordination failures so that bilingual speakers use only $B$ when they interact between them, is what is needed to keep alive a threatened language. But $\left(s_{\mathbf{2}}, s_{\mathbf{1}}\right)$ and $\left(s_{\mathbf{1}}, s_{\mathbf{2}}\right)$ are weak because they are not evolutionary stable nor dynamically stable. On the other hand, $(x, x)=\left(1-\frac{c(1-\alpha)}{\alpha m}, 1-\frac{c(1-\alpha)}{\alpha m}\right)$ is strong due to its stability properties, but weak in terms of language diversity (the combined effect of imperfect information, politeness norms, and other factors such as scarce formal and informal usages of the language, and less developed discursive models, could reduce the use of B in this equilibrium to a very low level).

Monolingual equilibria of the type $\left(s_{\mathbf{2}}, s_{\mathbf{1}}\right)$ and $\left(s_{\mathbf{1}}, s_{\mathbf{2}}\right)$ would typically occur in geographical areas where the speech community of $\mathrm{B}$ is relatively important. These are places where agents interact continuously so that they know well each other, and it is common knowledge who speaks $B$. Now, let us introduce a small perturbation; for instance, let the members of these communities move away to other areas so that they must interact with peo- 
ple unknown to them. Then our model predicts that the bilingual agents would tend to play instead of the above profiles, those of the mixed strategy equilibrium; revealing in this manner the unstable nature of the monolingual equilibria $\left(s_{\mathbf{2}}, s_{\mathbf{1}}\right)$ and $\left(s_{\mathbf{1}}, s_{\mathbf{2}}\right)$.

\section{Conclusions}

The Language Game makes explicit the position of bilingual agents who ought to make language choices continuously under imperfect knowledge.

The contact between $\mathrm{A}$ and $\mathrm{B}$ produces a negative externality upon the use of the minority language $B$. This is illustrated by the situation where two bilingual players meet and fail to coordinate in the minority language $B$, because they are not informed about the bilingual nature of each other. We show that this may happen even if bilingual agents are willing to speak in $B$ and have payoff incentives to do so. Thus, the imperfect information reduces considerably the use of the minority language, aggravating its minority status.

Nevertheless, there is a bilingual bayesian-perfect equilibrium where the bilingual agents use both $A$ and $B$. The equilibrium is shown to be robust: evolutionary stable and asymptotically stable for the single population replicator dynamics. But, at the same time, this equilibrium, as language convention, provides a weak support to language diversity. The use that bilingual speakers make of $\mathrm{B}$ in this equilibrium depends on the imperfect information, politeness norms and other factors outside the model, (such as the scarcity of formal and informal usages of the minority language and, hardly developed oral and written discursive models) that would reduce the use of $B$ to very low levels. That would the outcome of a widely used $(A B, B A)$ or Hawk-Hawk strategy combination.

A straightforward lesson we can learn from this paper is that eliminating the imperfect information structure of the language game will improve considerably the use of the minority language among the bilingual players. It is fair to say that, in some real life bilingual settings, even in non-familiar ones, one might detect signals, such as a certain accent or specific physical looks, that might help deducing who is bilingual and who is not. This can ensure the use of $B$ and help to maintaining the linguistic diversity of the society. But it should be taken into account that a language policy consisting of marking or labelling people to denote their bilingual nature could be an additional source of conflict. 
The models shows that the cost $c$ (of being forced to change your chosen language) could be a curse for bilingual individuals' coordination in $B$. The reduction of $c$ could be one of the language policy targets: by designing policies to promote the use of $B$, or to make people aware of some wrong notions of politeness when choosing language strategies.

Given the difficulty to measure the use bilingual players make of $B$ in anonymous situations, we believe experimental work is warranted. There are many questions of interest. In particular, it would be interesting to compare loaded and unloaded information treatments within a bilingual society. That

is, comparing individual behavior in the Language Game, where actions $A$ and $B$ have no further meaning with the behavior where $A$ and $B$ represent the majority and minority languages. It will also be interesting to know the speech conventions that appear in an experimental setting and compare with the prediction made in Proposition 2. Also, the influence of politeness in the strategies used, and the gender differences of that influence. We leave those questions for further research.

\section{References}

Abrams D.M. and Strogatz, S.H. (2003) "Modeling the Dynamics of Language Death" Nature 424, 900.

Annamalai, E.(2000). "The Linguistic Heritage of India" in Linguistic Heritage of India and Asia (O.N. Koul and L. Devaki Eds.), Manasagangotri, Mysore, India: Central Institute of Indian Languages.

Binmore, K. Gale, J. and Samuelson, L.(1999). "Learning to be Imperfect: the Ultimatum Game" Games and Economic Behavior 8:56-90.

Brown, P. and Levinson, S. C. (1987) Politeness: Some Universals in Language Usage (Studies in Interactional Sociolinguistics). Cambridge: CUP.

Cabrales, A. (2000) "Stochastic Replicator Dynamics" International Economic Review 41: 451-481.

Cabrales, A. and Uriarte, J.R. (2010) "Doubts and Equilibria" Mimeo

Castelló, X., Eguiluz, V. M. and San Miguel, M. (2006) "Ordering Dynamics with Two Non-Excluding Options: Bilingualism in Language Competition" New Journal of Physics 8.

Castelló, X., Toivonen, R., Eguiluz, V. M., Xaramaki, J., Kaski, K. and San Miguel, M. (2007) "Anomalous Life Time Distributions and Topological Traps in Ordering Dynamics"Europhysics Letters 79, 66066 
Crystal, D. (1987). The Cambridge Encyclopedia of Language, Cambridge, UK: Cambridge University Press.

Crystal, D. (2001). Language Death, Cambridge, UK: Cambridge University Press.

E.J.-G.V. (2009). IV Mapa Soziolinguistikoa

Fishman, J. A.(1991). Reversing Language Shift: Theoretical and Empirical Foundations of Assistance to Threatened Languages, Clevedon: Multilingual Matters.

Fishman, J. A.(2001). "Why is it so Hard to Save a Threatened Language?" in J. A. Fishman (Ed.) Can Threatened Languages be Saved? Clevedon: Multilingual Matters.

E. Goffman (1967). Interaction Ritual. Harmondsworth: Penguin.

Grin, F. (1996). "The Economics of Language: Survey, Assessment and Prospects", (dir.) International Journal of the Sociology of Language, 121: $17-44$.

Grin, F. and Vaillancourt, F. (1999). "The Cost-Effectiveness Evaluation of Minority Language Policies: Case Studies on Wales, Ireland and the Basque Country", Monograph Series, No 2, Flensburg: European Centre for Minority Issues.

Grin, F. (2003). "Language Planning and Economics", Current Issues in Language Planning, 4, No 1: 1-66.

Krauss, M. (1992). "The Scope of the Language Endangerment Crisis and Recent Response to it" in Studies in Endangered Languages (K. Matsumura Ed. (1998)). Tokio: Hituzi Syobo.

Lakoff, R. (1973) "The logic of politeness; or mind your p's and q's", Papers from the 9th regional meeting of the Chicago Linguistic Society. Chicago: Chicago Linguistic Society 292-305

Mira, J.B. and Paredes, A. (2005) "Interlinguistic Similarity and Language Death Dynamics" Europhysics Letters 69 (6) 1031-1034.

Nelde, P. (1997). "Language Conflict" in F. Coulmas (ed.) The Handbook of Sociolinguistics, London: Blackwell 285-300.

Patriarca, M. and Leppänen, T. (2004) "Modeling Language Competition" Physica A 338 (1-2), 296-299.

Pinasco, J.P. and Romanelli (2006) "Coexistance of Languages is Possible" Physica A 361, 355-360.

Pool, J. (1986). "Optimal Strategies in Linguistic Games" in The Fergusonian Impact Vol.2: Sociolinguistics and the Sociology of Language (Fishman, J. et al. Editors). Berlin-New York-Amsterdan: Mouton de Gruyter. 
Schlag, K. (1998) "Why Imitate, and if so, How? A Boundedly Rational Approach to Multi-Armed Bandits", Journal of Economic Theory 78, 130156.

Selten, R. and Pool, J. (1991). "The Distribution of Foreing Language Skills as a Game Equilibrium" in R. Selten (Ed.) Game Equilibrium Models, vol. 4 Berlin: Springer-Verlag, 64-84.

Stauffer, D. and Schulze, Ch. (2005) "Microscopic and macroscopic Simulation of Competition between Languages" Physics of Life Review 2: 89-116.

Stauffer, D., Castelló, X. Eguiluz, V.M. and San Miguel, M. (2007) Microscopic Abrams-Strogatz Model of Language Competition" Physica A, 374: 835-842.

Uriarte, J.R. (2006). "A Behavioral Foundation for Models of Evolutionary Drift", Journal of Economic Behavior and Organization vol. 63, Issue 3, July 2007, 497-513.

Vaillancourt, F. (1996). "Language and Socioeconomic Status in Quebec: Measurement, Findings Determinants and Policy Costs", International Journal of the Sociology of Language 121, 69-92.

Wang, W. S.-Y. and Minnet, J. W. (2005) "The Invasion of Language. Emergence, Change, and Death" Trends in Ecology and Evolution 20 (5):263269.

Wang, W. S.-Y. and Minnet, J. W. (2008) "Modelling Endangered Languages: The Effects of Bilingualism and Social Structures" Lingua 118:19-45.

Weibull, J.W. (1995) Evolutionary Game Theory, Cambridge, Mass.: the MIT Press

Wickström, B.-A. (2005). "Can Bilingualism be Dynamically Stable?", Rationality and Society 17, 81-115.

Wurm, S.A. (2001). Atlas of the World's Languages in Danger of Disappearing, Paris-Camberra: UNESCO Publishing.

\section{Appendix}

First, let us describe each player's expected payoffs in each information set. Player I's expected payoff at Bilingual Ia information set is the following:

$E_{I}($ Bilingual $I a)=\alpha(1-p)\{q[r m+(1-r) n]+(1-q) n\}+(1-\alpha)(1-$ p) $n+\alpha p[s m$

$+(1-s)(n-c)]+(1-\alpha) p(n-c)$

Therefore, 


$$
E_{I}(\text { Bilingual Ia })=\left\{\begin{array}{cl}
\alpha q r(m-n)+n & \text { if } p=0 \\
\alpha s(m-n)+n-c(1-\alpha s) & \text { if } p=1
\end{array}\right.
$$

The probability of reaching player II's Majority Language $(M a L)$ information set is $\alpha^{2}(1-p)+(1-\alpha) \alpha$. Hence, player II's Bayes consistent beliefs at nodes $x$ and $y$ of $M a L$ information set are $\mu_{I I}(x)=\alpha(1-p) /(1-\alpha p)$ and $\mu_{I I}(y)=(1-\alpha) /(1-\alpha p)$, respectively. Thus, the expected payoff of player II at this information set is given by the following expression:

$$
E_{I I}(M a L)=\frac{\alpha(1-p)}{1-\alpha p}\{q[(1-r)(n-c)+r m]+(1-q) n\}+\frac{1-\alpha}{1-\alpha p}(n-q c)
$$

Therefore,

$$
E_{I I}(M a L)=\left\{\begin{array}{cc}
n & \text { if } q=0 \\
\frac{1}{1-\alpha p}[\alpha r(m-n)-c(1-\alpha r)-\alpha p r(m-n) & \\
-\alpha p r c+\alpha p(c-n)+n] & \text { if } q=1
\end{array}\right.
$$

Recall that player I's pure strategy set is $S_{I}=\{B B, B A, A B, A A\}$. Since $B B$ and $B A$ are equivalent, we shall refer to them as $B B$, hence $S_{I}=$ $\{B B, A B, A A\}$.

\section{Proof of Result 1}

Formally, when $r=1$, (thus, player I chooses $B$ in the subgame starting at Bilingual $I b$ information set), (2) becomes

$$
E_{I I}(M a L)=\left\{\begin{array}{cl}
n & \text { if } q=0 \\
\frac{1}{1-\alpha p}[n-\alpha p m] & \text { if } q=1
\end{array}\right.
$$

If $p=0$ and $r=1$, and this means that player I's strategy is $A B$, then $E_{I I}(M a L)=n$, for all $q, s=[0,1]$. In other words, if player I starts the conversation using language $A$ and is planning to switch to $B$ at Bilingual $I b$, then player II is made to be indifferent between $A$ and $B$ in his two information sets, which is to say that he is indifferent between all his pure strategies.

When $s=1$, meaning that player II chooses $B$ in the subgame starting at $M i L$ informations set, then (1) becomes

$$
E_{I}(\text { Bilingual } I a)=\left\{\begin{array}{cl}
\alpha q r(m-n)+n & \text { if } p=0 \\
n & \text { if } p=1
\end{array}\right.
$$

We can see that if $s=1$ and $q=0$, -that is, if player II's strategy is $B A$-, then $E_{I}($ Bilingual $I a)=n$ for all $p, r=[0,1]$. Hence, player $\mathrm{I}$ is indifferent between the actions available at Bilingual Ia information set as well as those available at Bilingual $I b$ information set. This means that Player I is indifferent between all his pure strategies when player II chooses $s=1$ and $q=0$. Therefore, we have found a strategy profile that is an equilibrium $(A B, B A)$, which is also Bayesian perfect. The behaviour 
strategy profile $\pi=\left(\pi_{I}, \pi_{I I}\right)=(p=0, r=1 ; s=1, q=0)$ and player II's belief system $\mu=\left(\mu_{I I}(x)=\alpha\right.$ and $\left.\mu_{I I}(y)=1-\alpha\right)$ is a Bayesian perfect equilibrium for the Language Game. In this equilibrium the spoken language between bilingual players is $A$. If we keep $p=0$ and $q=0$, then the Bilingual $\mathrm{Ib}$ and Minority Language information sets are not reached with positive probability. Hence, combining the values 0 and 1 assigned to $r$ and $s$, we would get Nash equilibria that are not Bayesian perfect, such as $(A B, A A)(A A, B A)$ and the pooling equilibrium $(A A, A A)$. Note that in all these equilibria, the language spoken among the bilingual players is $A$. The Bayesian perfect equilibrium $(A B, B A)$ might be viewed as a language coordination failure because bilingual players do intend to use the language they prefer most, but they fail and end up using $A$.

Hence, any departure from $p=0, r=1, q=0, s=1$ that permits the realization of the desired coordination in language $B$ would be an equilibrium too because no player would get a lower payoff and at least one player would get a higher payoff. These are the following cases:

(i) If $p=1, r=1$, then $E_{I I}(M a L)=\left\{\begin{array}{cl}n & \text { if } q=0 \\ n-c & \text { if } q=1\end{array}\right.$; therefore $q=0$ is the best choice and at $M i L$, since player I has revealed his bilingual nature by choosing $p=1$, then player II must choose $s=1$. Against $s=1$, $q=0$ (which means, against player II's $B A$ ), we have seen that player I is indifferent between any $p, r=[0,1]$; thus $p=1, r=1$ is a best reply. Hence, $(B B, B A)$ is a Bayesian perfect equilibrium (with $\pi=(p=1, r=1 ; s=1$, $q=0)$ and $\mu=\left(\mu_{I I}(x)=0\right.$ and $\left.\left.\mu_{I I}(y)=1\right)\right)$, in which the spoken language among the bilingual players is $B$.

(ii) If $q=1, s=0$, then $E_{I}$ (Bilingual Ia) $=\left\{\begin{array}{cl}\alpha r(m-n)+n & \text { if } p=0 \\ n-c & \text { if } p=1\end{array}\right.$. Hence $p=0$ is the best choice at Bilingual Ia and, since player II has revealed his type by choosing $q=1$, it must be $r=1$ at Bilingual Ib. Again, if $p=0$ and $r=1$ player II is indifferent among all his pure strategies, so $q=1$, $s=0$ is a best choice. Thus, we get the non Bayesian perfect equilibrium $(A B, A B)$ in which the spoken language among the bilingual players is $B$.

(iii) If $q=1, s=1$, then $E_{I}($ Bilingual $I a)=\left\{\begin{array}{cl}\alpha r(m-n)+n & \text { if } p=0 \\ n & \text { if } p=1\end{array}\right.$. Hence, the best choices are $p=0$ and $r=1$. Hence, $(A B, B B)$ is a Bayesian perfect equilibrium (with $\pi=(p=0, r=1 ; s=1, q=1)$ and $\mu=($ $\mu_{I I}(x)=\alpha$ and $\left.\mu_{I I}(y)=1-\alpha\right)$ ) in which the spoken language between bilingual players is $B$. 
Hence

$$
B P N^{1}=\{(A B, B A),(A B, B B),(B B, B A)\}
$$

In $(A B, B B)$ and $(B B, B A)$ the interaction among bilingual players occurs in $B$, but involve the play of weakly dominated strategies. Whereas, the strategies in $(A B, B A)$ are dominant for both players, and the interaction occurs in $A$. Note that player I's $A B$ strategy is a best response against all strategies of player II, although it is not the only best response against some strategies. Hence, $A B$ is a weakly dominant strategy for player I. This is so because a bilingual player I who uses this strategy starts the conversation with $A$ and with this choice he will avoid to suffer the frustration cost, $c$. Moreover, if she is matched to a bilingual player II, who reveals her bilingual nature by choosing $B$ at $M a L$ information set (i.e. a player II using either $B B$ or $A B)$, player I then switches to $B$ at Bilingual $I b$ information set and gets the maximum payoff of $m$. For player II, $B A$ is a best response against all player I's strategies (against some strategies it is not the only one though). Thus, $B A$ is a weakly dominant strategy for player II, because to a bilingual player I who reveals her bilingual nature by beginning the conversation using $B$ (when using $B B$ or the equivalent strategy $B A$ ) he will answer with $B$ and if player I starts the conversation using $A$ he replies with $A$. Therefore, the use of $A B$ for player I and the use of $B A$ for player II, allows both players to avoid the minimum payoff $(n-c)$ and in principle to reach the maximum payoff of $m$.

\section{Proof of Result 2}

Let us assume that $p=0$, then from (2)

$$
E_{I I}(M a L)=\left\{\begin{array}{cl}
n & \text { if } q=0 \\
\alpha r(m-n)-c(1-\alpha r)+n & \text { if } q=1
\end{array}\right.
$$

Under the conditions of Case 2, given $p=0$ and any $r \in[0,1],-$ which means that we are considering player I's pure strategies $A B$ and $A A-, q=$ 0 maximizes $E_{I I}(M a L)$. Note that this result is independent of any $s \in$ $[0,1]$, therefore, player II will play the (strictly undominated) $B A$ and $A A$ strategies.

Let us assume now that $q=0$, then from (1)

$E_{I}($ Bilingual Ia $)=\left\{\begin{array}{cl}n & \text { if } p=0 \\ \alpha s(m-n)-c(1-\alpha s)+n & \text { if } p=1\end{array}\right.$

Therefore, given $q=0$ and any $s \in[0,1]$, - which means that we 
are considering player II's pure strategies $B A$ and $A A-, p=0$ maximizes $E_{I}$ (Bilingual Ia). This result is independent of the value of $r \in[0,1]$. Therefore, player I will play the (strictly undominated) $A B$ and $A A$ strategies. Thus, $B P N^{2}=\{(A B, B A)\}$

\section{Proof of Result 3}

Let us assume that $p=0$ and $r=1$ (in other words, assume player I's $A B$ strategy), then from (2)

$$
E_{I I}(M a L)=\left\{\begin{array}{lr}
n & \text { if } q=0 \\
\alpha(m-n)+n-c(1-\alpha) & \text { if } q=1
\end{array}\right.
$$

Given the payoff assumption in Regime $3, q=1$ maximizes $E_{I I}(M a L)$ given any $s \in[0,1]$ (thus against player I's $A B$, player II's best responses are $B B$ and $B A$.

Now suppose that $q=1$, then

$$
E_{I}(\text { Bilingual Ia })=\left\{\begin{array}{cl}
\alpha r(m-n)+n & \text { if } p=0 \\
\alpha s(m-n)-c(1-\alpha s)+n & \text { if } p=1
\end{array}\right.
$$

When $p=0$ and $r=1$ the expected payoff for player I at Bilingual Ia is maximized when $q=0$ and any $s \in[0,1]$. Hence, against player II's $A B$ and $B B$ strategies, player Is best response is $A B$. When both player I and II choose $B$ at Bilingual $I b$ and Minority Language informations sets, the strategy profile $(A B, B B)$ would be a Bayesian perfect equilibrium (that is, the behaviour strategy profile $\pi=\left(\pi_{I}, \pi_{I I}\right)=(p=0, r=1, s=1, q=1)$ and player II's belief system $\mu=\left(\mu_{I I}(x)=\alpha\right.$ and $\left.\mu_{I I}(y)=1-\alpha\right)$ is a Bayesian perfect equilibrium of the Language Game). ( $A B, A B)$ is a Nash equilibrium, but not Bayesian perfect. In both equilibria the language spoken by the bilingual players is $B$.

Suppose now that bilingual player I chooses $p=1$ and $r=1$ (that is, player I chooses $B B)$. Then, $E_{I I}(M a L)=n-c q$. Hence, $E_{I I}(M a L)$ is maximized when $q=0$ and any $s \in[0,1]$. Now, if $q=0$ and $s=1$, then from (1) and given the payoffs in Case 3 we have $E_{I}$ (Bilingual $\left.I a\right)=$ $\alpha(m-n)+n-c(1-\alpha)$ if $p=1$ and any $r \in[0,1]$. Then $(B B, B A)$ is a Bayesian perfect equilibrium (where now $\pi=\left(\pi_{I}, \pi_{I I}\right)=(p=1, r=1$, $s=1, q=0)$ and $\mu=\left(\mu_{I I}(x)=0\right.$ and $\left.\left.\mu_{I I}(y)=1\right)\right)$. In this equilibrium the language spoken by the bilingual players is $B$ as well. 


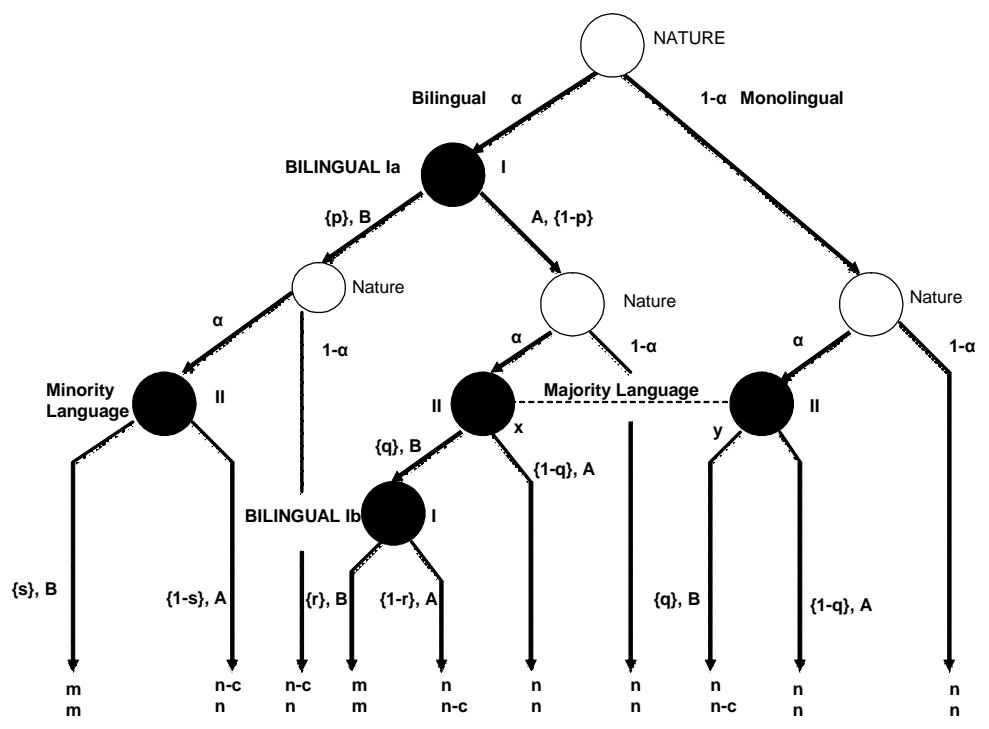

